Report

\title{
Safety issues with cyclosporine
}

\author{
Charles N. Ellis, MD
}

From the Department of Dermatology, University of Michigan Medical School, Ann Arbor, Michigan

\section{Correspondence}

Charles N. Ellis, MD, Department of Dermatology, University of Michigan Medical School, 1910 A. Alfred Taubman Health Care Center, Ann

Arbor, MI 48109-0314

The more even absorption characteristics of the Neoral ${ }^{(1)}$ formulation of cyclosporine contribute to its improved safety profile when compared with Sandimmune ${ }^{\circledR}$. Excessive dose adjustments of cyclosporine are reduced, stable absorption is offered, and variability in cyclosporine blood levels is avoided. Short-term use of Neoral, i.e. for treatment up to I year, also enhances its overall safety.

As discussed in other articles in this supplement, the potential for side-effects of Neoral may be reduced by careful patient selection and by proper dosing. The adverse effects that may be associated with appropriate dosing of Neoral are reviewed in this article.

\section{Early vs persistent symptomatic adverse events}

The symptomatic adverse reactions that occur with cyclosporine therapy fall into two types. First are those reactions that occur in a concentration-dependent fashion during initial therapy, including gastrointestina! distress, fatigue, headache, malaise, and joint or muscle discomfort. These reactions typically resolve without treatment after several weeks. Tremors and paresthesias have been reported, although they occur infrequently at doses of cyclosporine recommended for dermatologic conditions. When present, the symptoms are likely to improve with time during therapy.

Second are the more persistent, clinically evident reactions that include hypertension, hirsutism, and gingival hyperplasia.

The overall risk of developing hypertension while on cyclosporine therapy is variable. As would be expected, the risk is greater in older patients and in those who have higher baseline blood pressures. Persistent hypertension, i.e. blood pressure greater than $140 / 90 \mathrm{~mm}$
$\mathrm{Hg}$, can often be managed with salt restriction, exercise, and fish oil; antihypertensive drug therapy is used when other measures fail.

The incidence of drug-induced hirsutism is $\approx 25 \%$ within the first 4 months of treatment.' The problem appears to involve primarily terminal hair. Gingival hyperplasia occurs in $\approx 10 \%-15 \%$ of patients ${ }^{1}$ and can be ameliorated at least in part by careful dental hygiene.

Table I lists the reasons why patients in clinical trials at our center discontinued therapy over an extended treatment period of 3 years. Eighty-five parients began the study. After $\approx 1,2$, and 3 years, 64,27 , and 16 patients, respectively, remained in the protocol. Currently, treatment periods of I year or less are recommended.

\section{Serious adverse effects}

\section{Laboratory values}

The most common changes in laboratory test values associated with Neoral are shown in Table 2. Increases in uric acid or bilirubin are not generally of concern in patients who are receiving the doses of Neoral appropriate to dermatologic conditions. Hyperlipidemia induced by cyclosporine is usually mild and often can be managed by dietary changes, by increased physical activity, or, when necessary, by pharmacologic intervention. Replacement magnesium therapy may be necessary in some patients.

\section{Renal toxicity}

The primary concern with the recommended use of Neoral is the potential for toxic effects on the kidney. ${ }^{2-}$ 4 The early and reversible vasoconstrictive activity of the drug may precipitate a rise in serum creatinine concentrations within the first few weeks of therapy. ${ }^{1}$ After at least I year of treatment, cyclosporine therapy 
Table 1 Reasons for stopping cyclosporine therapy during 3-year, single-center clinical trial*

\begin{tabular}{lc}
\hline Category† & Percentage of patients who left study $(N=69)$ \\
Patient violated protocol, moved away, or miscellaneous reasons & 33 \\
Kidney-related side-effectsł & 33 \\
Unrelated illnesses & 14 \\
Cardiovascular side-effects§ & 10 \\
Lack of efficacy of cyclosporine & 4 \\
Entered long-term remission during cyclosporine therapy & 4 \\
Total & 981 \\
\hline
\end{tabular}

*The current recommendation is to treat for periods of I year or less.

tThe author has combined various reasons for leaving the study into these categories.

†ncludes elevations in serum creatinine or urea nitrogen, decline in glomcrular filtration rate, renal biopsy evidence of fibrosis, and patients in whom concern was raised about their requirement for concurrent use of nephrotoxic agents such as nonsteroidal anti-inflammatory drugs.

\$ncludes hypertention, migraines, and other heart or vascular conditions.

IDoes not total too because of rounding.

Table 2 Laboratory changes associated with Neoral therapy

\begin{tabular}{ll}
\hline Increased values & Decreased values \\
\hdashline Creatinine & Glomerular filtration rate \\
Urea nitrogen (BUN) & Magnesium \\
Cholesterol/triglycerides & \\
Bilirubin & \\
Uric acid & \\
\hline
\end{tabular}

is also associated with tubular atrophy and interstitial fibrosis..$^{5-7}$ None of the patients in our clinical trials, including those receiving treatment for as long as 3 years, required treatment for kidney disorders. The long-term potential for progressive renal failure requires further clarification, however, as does the impact of other variables, such as ageing or the use of such agents as nonsteroidal anti-inflammatory drugs on declining renal function.

What are the clinical implications with regard to renal toxicity? In general, the potential for toxicity can be minimized by doses that do not exceed $5 \mathrm{mg} / \mathrm{kg}$ per day of either Ncoral or Sandimmune, and by dosage reduction in the event that serum creatinine rises more than $30 \%$ above baseline values. Fish oil supplements have been suggested to minimize renal toxicity. ${ }^{8,9}$ Measurement of glomerular filtration rate may be useful in monitoring renal function in patients with pre-existing risk factors for kidney disease or in patients receiving cyclosporine beyond the recommended time course. The use of short-term therapy would also be expected to avoid the occurrence of irreversible renal dysfunction.

\section{Cancer}

The development of cancer as a result of cyclosporine therapy is an extremely rare phenomenon, ${ }^{10}$ particularly when compared with the more straightforward relationship between the drug and renal side-effects. The occurrence of lymphoma or other cancers more likely reflects the degree of deep immunosuppression induced by this class of agents, but this is not as great a concern with the treatment of psoriasis, where cyclosporine is administered in doses below $5 \mathrm{mg} / \mathrm{kg}$ per day for intermittent periods, and without other immunosuppressive agents.

\section{Cutaneous side-effects}

Infections such as warts, impetigo, and tinea are of concern in transplant patients receiving cyclosporine; however, this is not a substantive problem in patients receiving low doses for treatment of psoriasis.

Rare side-effects of Neoral include isolated case reports of severc acne, folliculitis, and reversible benign lymphocytic infiltrates. ${ }^{\mathrm{O}, \mathrm{II}}$

\section{Monitoring guidelines}

Table 3 contains monitoring recommendations prior to and during Neoral therapy. An accurate bascline serum creatinine measurement is important in order to assess 
Table 3 Recommendations for monitoring prior to and during Neoral therapy

\begin{tabular}{|c|c|}
\hline Parameter & Action \\
\hline \multicolumn{2}{|l|}{ Prior to therapy } \\
\hline Serum creatinine & $\begin{array}{l}\text { Obtain at least two measurements within } \\
10 \% \text { of each other to determine true } \\
\text { baseline level }\end{array}$ \\
\hline \multicolumn{2}{|c|}{ At baseline, after 2 and 4 weeks, and then at least monthly } \\
\hline Blood pressure & $\begin{array}{l}\text { Treat hypertension or reduce cyclosporine } \\
\text { dose if possible }\end{array}$ \\
\hline Creatinine & $\begin{array}{l}\text { Reduce cyclosporine dose if value }>30 \% \\
\text { over baseline }\end{array}$ \\
\hline Urea nitrogen & $\begin{array}{l}\text { Assess kidney function further if significantly } \\
\text { and persistently abnormal }\end{array}$ \\
\hline Lipids & Treat if significantly abnormal \\
\hline Bilirubin, liver function & $\begin{array}{l}\text { Expect increases, but dose reduction } \\
\text { usually not indicated in bilirubin }\end{array}$ \\
\hline Uric acid & Treat if significantly abnormal \\
\hline Magnesium & Provide replacement therapy if low \\
\hline Electrolytes & Treat if significantly abnormal \\
\hline New medications & $\begin{array}{l}\text { Determine if new medication affects or is } \\
\text { affected by cyclosporine metabolism }\end{array}$ \\
\hline
\end{tabular}

Adapted from Ellis CN, Cyclosporine in the treatment of severe psoriasis. In: Weinstein GD and Gottleib AB (eds). Therapy of Moderate-to-Severe Psoriasis. Portland, Ore: The National Psoriasis Foundation, I993: I 5.

changes during therapy, and two separate baseline tests within ro\% of each other help overcome procedural inaccuracies.

Out-patient urine creatinine clearance tests tend to be unreliable. Creatinine clearance can be calculated from formulas based on serum creatinine but, for many patients, adds little practical information. Glomerular filtration rates are probably unnecessary in most patients when cyclosporine is used for the treatment of psoriasis as recommended.

Blood pressure should be followed every 2 weeks for the first 4 weeks' of therapy, and monthly thereafter. It is not usually necessary to track blood levels of cyclosporine routinely, but measurement may be helpful in the rare patient who does not experience any clinical improvement on therapy. In this case, the cyclosporine blood level may differentiate a true nonresponder from a patient experiencing poor absorption. Because many laboratories use transplantation indications as their reference for normal levels of cyclosporine, the reported laboratory "normal range" may not be helptul.

\section{The future literature on Neoral}

Now that Neoral is approved by the Food and Drug Administration for use in psoriasis, it is likely that there will be a substantial increase in usage. This will likely lead to a number of reports of side-effects published by dermatologists in the dermatologic literature. Readers of these reports should recognize that many of the sideeffects will occur once or rarely. Further, readers should not expect to see a balancing number of articles on the efficacy of cyclosporine because the results of cyclosporine therapy in many of the responsive disorders have already been published.

\section{Conclusions}

The major organ at risk during cyclosporine therapy is the kidney; however, risk to renal function can be reduced by attention to dosing and routine measures of kidney function, specifically scrum creatinine. When used within the guidelines and for periods of $x$ year or less, cyclosporine, particularly the Neoral formulation, may provide substantial benefit to patients with psoriasis and other dermatologic conditions. Neoral is a formulation of cyclosporine that provides consistent and predictable absorption.

\section{References}

I Ellis CN, Fradin MS, Messana JM, et al. Cyclosporine for plaque-type psoriasis. Results of a multidose, double-blind trial. $N$ Engl J Med x991; 324: 277-28 4 .

2 Powles AV, Carmichael D, Hulme B, ct al. Renal function after long-term low-dose cyclosporin for psoriasis. Br J Dematol r990; 122: 665-669.

3 Messana JM, Rocher LL, Ellis CN, et al. Fifects of cyclosporine on renal function in psoriasis patients. I Am Acad Dermatol r990; 23: $1288-1293$.

4 Feutren G, Mihatsch MG. Risk factors for cyclosporine-induced nephropathy in patients with autoimmunc diseases. N Engl / Med 1992; 326: 16.54I. 660 .

5 Mihatsch MJ, Wolff K. Consensus conference on cyclosporine A for psoriasis, February 1992. Br I Dermatol 1992; 126: 621-623.

6 Zachariae H, Hansen HE, Kragballe K, et al. Morphologic renal changes during cyclosporine treatment of psoriasis. I An Acad Dematol 1992; 26 : 4. $5-419$.

7 Young EW, Ellis CN, Messina JM, et al. A prospective study of renal structure and function in psoriasis patients treated with cyclosporine. Kidncy lnt 1994: 46: $1216-1222$.

8 Homan van der Heide JJ, Bilo H], Donker JM, ot al. Effect of dictary tish oil on renal function and 
rejection in cyclosporine-recipients of renal transplants. $N$ Engl I Med I993; 329: 769-773.

9 Stoof TH, Korstanje MJ, Bilo HF, et al. Does fish oil protect renal function in cyclosporine-treated psoriasis patients? J Intern Med I989; 226: 437-44I.

to Fradin MS, Ellis CN, Voorhees JJ. Management of patients and side effects during cyclosporine therapy for cutaneous disorders. I Am Acad Dernatol 1990; 23: $1265-1275$.

It Gupta AK, Cooper KD, Ellis CN, et al. Lymphocytic infiltrates of the skin in association with cyclosporine therapy. I Am Acad Dermatol I990; 23: II 37-II4I. 
This document is a scanned copy of a printed document. No warranty is given about the accuracy of the copy. Users should refer to the original published version of the material. 